\title{
A CRISPR/Cas9 Cleavage System for Capturing Fungal Secondary Metabolite Gene Clusters
}

\author{
Xinran $\mathrm{Xu}^{1,2}$, Jin Feng ${ }^{1}$, Peng Zhang ${ }^{1}$, Jie Fan ${ }^{1}$, and Wen-Bing Yin ${ }^{1,2 *}$ \\ 'State Key Laboratory of Mycology and CAS Key Laboratory of Microbial Physiological and Metabolic Engineering, \\ Institute of Microbiology, Chinese Academy of Sciences, Beijing 100101, P.R. China \\ ${ }^{2}$ Savaid Medical School, University of Chinese Academy of Sciences, Beijing 100049, P.R. China
}

\begin{abstract}
More and more available fungal genome sequence data reveal a large amount of secondary metabolite (SM) biosynthetic 'dark matter' to be discovered. Heterogeneous expression is one of the most effective approaches to exploit these novel natural products, but it is limited by having to clone entire biosynthetic gene clusters (BGCs) without errors. So far, few effective technologies have been developed to manipulate the specific large DNA fragments in filamentous fungi. Here, we developed a fungal BGC-capturing system based on CRISPR/Cas9 cleavage in vitro. In our system, Cas9 protein was purified and CRISPR guide sequences in combination with in vivo yeast assembly were rationally designed. Using targeted cleavages of plasmid DNAs with linear (8.5 kb) or circular (8.5 kb and $28 \mathrm{~kb}$ ) states, we were able to cleave the plasmids precisely, demonstrating the high efficiency of this system. Furthermore, we successfully captured the entire Nrc gene cluster from the genomic DNA of Neosartorya fischeri. Our results provide an easy and efficient approach to manipulate fungal genomic DNA based on the in vitro application of Cas9 endonuclease. Our methodology will lay a foundation for capturing entire groups of BGCs in filamentous fungi and accelerate fungal SMs mining.
\end{abstract}

Keywords: CRISPR/Cas9, secondary metabolites, heterogeneous expression, biosynthetic gene clusters, filamentous fungi

Received: August 20, 2020 Accepted: October 26, 2020

First published online: October 30, 2020

*Corresponding author Phone: +86-10-64806170 E-mail: yinwb@im.ac.cn

Supplementary data for this paper are available on-line only at http://jmb.or.kr.

pISSN 1017-7825 eISSN 1738-8872

Copyright(C) 2021 by The Korean Society for Microbiology and Biotechnology

\section{Introduction}

Fungal secondary metabolites (SMs) exhibit diverse bioactivities and have been applied widely in different fields including industry, agriculture and medicine [1]. In recent years, a vast number of biosynthetic gene clusters (BGCs) have been found by high-throughput genome sequencing [2, 3]. However, most of the gene clusters are transcriptionally inactive, i.e. 'silent', under standard laboratory or naturally observed cultivation [3-5]. To activate these 'silent' BGCs, genome mining strategies either by cloning and expressing the entire group of BGCs in heterologous hosts or manipulating regulators directly in native hosts have been developed for novel metabolite discovery and their biosynthetic analysis [6]. So far, heterologous clusters are commonly expressed in fungi, especially in engineered Aspergillus species and Saccharomyces cerevisiae [7, 8]. A number of compounds, such as asperfuranone [9], geodin [10], neosartoricin B [11], citreoviridin [12] and aspercryptin [13] have been discovered by heterologous expression.

Even so, in vitro isolation and cloning of giant DNA fragments have remained key technical challenges in molecular biology due to the large size of fungal 'silent' BGCs (10 to $130 \mathrm{~kb}$ ) [14]. To obtain large fragments from native strains, transformation-associated recombination (TAR)-cloning has been utilized to manipulate fulllength genes with fragment size up to $250 \mathrm{~kb}$ based on in vivo homologous recombination in S. cerevisiae [15]. This strategy requires considerable effort and time to screen hundreds of colonies, but leads to low efficiency and specificity [16]. Notably, the TAR-cloning strategy has not been successful with DNA fragment assemblies in fungi. On the other hand, fungal artificial chromosome (FAC) systems were reported to shuttle and stably maintain large DNA fragments $(>150 \mathrm{~kb})$ in both Escherichia coli and filamentous fungi. The method utilizes unbiased random shear bacterial artificial chromosome (BAC) technology coupled with an autonomous fungal replicating element AMA1, and miscellaneous selection toward target fragments [14, 17]. However, it is laborious due to the amounts of colony screening and also costs a lot for colony sequencing.

Instead, the clustered regularly interspaced short palindromic repeats-associated protein (CRISPR/Cas) system from Streptococcus pyogenes has been used in numerous model organisms recently to edit the genome with high efficiency, versatility and specificity in vivo $[18,19]$. Firstly, an effector complex is assembled between the Cas 9 system with mature single guide RNA (sgRNA), which comprises CRISPR RNA (crRNA) and trans-activating 
crRNA (tracrRNA)-derived sequences. Afterwards, this Cas9-sgRNA complex recognizes target DNA guided by 5' sgRNA and specifically cleaves double-stranded DNA (dsDNA) to complete the editing process [20]. Notably, TAR cloning or Gibson was combined with a CRISPR/Cas9 system for isolation and cloning of large genomic sequences from human or bacteria more efficiently and specifically $[16,21,22]$. However, no methods have been reported yet to specifically capture an entire BGC in filamentous fungal genomes.

In this study, we present a CRISPR/Cas9 system in vitro for cleavage of fungal BGCs. The feasibility and efficiency of this system will be verified by cleaving plasmids with different shapes and sizes. Subsequently, the genomic DNA extracted from fungi will be cleaved by RNA-guided Cas9 endonuclease in vitro. The target gene clusters with large size will be inserted into vectors for heterogeneous expression. Compared to currently available methods, our method is a rapid approach to obtain target gene clusters accurately in filamentous fungal genomes.

\section{Materials and Methods}

\section{Strains and Plasmids}

E. coli Rosetta 2 (DE3) and E. coli EPI300 were purchased from Merck Millipore and Epicentre, respectively.

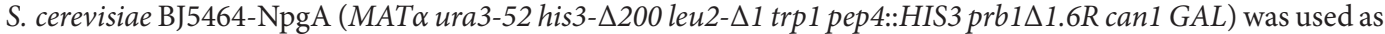
the host for homologous recombination. Fungal strains used in this study are Neosartorya fischeri and Pestalotiopsis fici. Plasmids are listed in Table 1 and oligonucleotide sequences are shown in Table S1.pX330 vector $[23,24]$ was used to obtain gene sequence of Cas 9 and used as target cleavage plasmid. pET-28a(+) vector was used to produce Cas9 protein. pCC1BAC vector was the starting vector of shuttle plasmids constructed in this study. Another target plasmid pYPZ37 (28.0 kb) harboring Pfma gene cluster was constructed in the previous study [25].

\section{Expression and Purification of Cas9}

The Cas9-expressing plasmid pYJF22 was generated as follows: a 4,104 bp-fragment containing Cas9 open reading frame (ORF) was obtained by PCR from pX330 with designed primers (Table S1) and inserted into the pET-28a(+) vector according to the ligation and cloning method to give the plasmid of pYJF22. The construct was then introduced into E. coli Rosetta 2 (DE3). The recombinant protein was purified as described previously [26].

\section{Synthesis and Purification of sgRNA}

CRSIPR design tool (http://crispr.mit.edu/) was used to identify CRISPR guide sequences. A double-stranded transcription template was prepared by amplifying a single-stranded oligonucleotide by PCR amplification of antisense template oligonucleotide with the T7 promoter sequence as a forward primer and the $3^{\prime}$ end of the antisense template as a reverse primer. Primers used for synthesis of sgRNA are sgRNA-F/R listed in Table S1. PCR reaction components are as follows: $4 \mu \mathrm{l}$ of $2.5 \mathrm{mM}$ dNTPs, $5 \mu \mathrm{l} 5 \times \mathrm{Q} 5$ reaction buffer, $0.5 \mu \mathrm{l}$ Q 5 High-fidelity DNA polymerase $(2 \mathrm{U} / \mu \mathrm{l}), 2.5 \mu \mathrm{l}$ target-specific primer X-sgRNA-P $(10 \mu \mathrm{M}), 1 \mu \mathrm{l}$ of universal primer sgRNA-F $(10 \mu \mathrm{M})$ and $2.5 \mu \mathrm{l}$ of universal primer sgRNA-R $(10 \mu \mathrm{M})$ without any template as well as sterile water to a total volume of $25 \mu \mathrm{l}$. This reaction was performed as follows: heat the reaction to $98^{\circ} \mathrm{C}$ for 30 second $(\mathrm{sec})$ to denature, then perform 30 cycles of PCR $\left[98^{\circ} \mathrm{C}\right.$ for $10 \mathrm{sec}$ (denaturation), $55^{\circ} \mathrm{C}$ for $20 \mathrm{sec}$ (annealing) and $72^{\circ} \mathrm{C}$ for $15 \mathrm{sec}$ (elongation)] followed by final elongation at $72^{\circ} \mathrm{C}$ for $2 \mathrm{~min}$ [27].

The synthesized DNA mentioned above was purified using DNA Clean \& Concentrator Kits (Zymo Research, USA). A concentration of 30-100 ng/ $\mu$ l purified DNA with $\mathrm{A}_{260 / 280}>1.8$ was measured by NanoDrop. Subsequently, in vitro transcription was performed using a T7 High Efficiency Transcription Kit (Transgen). After transcription, large quantities of RNA were purified by EasyPure RNA Purification Kit (Transgen) to give sgRNA. A concentration of 300-1,000 ng/ $\mu$ l synthesized sgRNA with $A_{260 / 280}>2.0$ was measured for purity detection by NanoDrop. Finally, synthesized sgRNA was diluted to a concentration of $300-1,000 \mathrm{nM}$ with RNase-free water for the preparation of Cas 9 cleavage assays.

\section{In Vitro Cas9 Endonuclease Cleavage Assays for Plasmids pX330 and pYPZ37}

Cas9-sgRNA complexes were constituted before cleavage by incubating Cas9 endonuclease with in vitro transcribed sgRNA in the reaction mixture containing $5 \times$ cleavage buffer (100 mM HEPES [pH 7.5], $500 \mathrm{mM}$ $\mathrm{KCl}, 25 \%$ glycerol, $5 \mathrm{mM} \mathrm{DTT}, 2.5 \mathrm{mM} \mathrm{EDTA}$ [pH 8.0], $10 \mathrm{mM} \mathrm{MgCl}_{2}$ ) and nuclease-free $\mathrm{H}_{2} \mathrm{O}$. After incubation at $30^{\circ} \mathrm{C}$ for $20 \mathrm{~min}$, the cleavage reaction was initiated by adding the target DNA to the reaction mixture immediately

Table 1. Plasmids used in this study.

\begin{tabular}{lll}
\hline Plasmid name & \multicolumn{1}{c}{ Description } & References \\
\hline pX330 & Streptococcus pyogenes Cas9, Amp & {$[23-24]$} \\
pYPZ37 & URA3, AfRibo, wA flanking, Amp, Pfma cluster (PfmaA-E, PfmaG, gpdA(p)::PfmaH) & {$[25]$} \\
pET-28a & Protein expression vector & Invitrogen \\
pCC1BAC & BAC vector & {$[21]$} \\
pYJF22 & S. pyogenes Cas9, Kan & This study \\
pYJF24 & URA3, PyrG, Cm, wA flanking in pCC1BAC- & This study \\
pYJF26 & URA3, AfRibo, Cm, wA flanking in pCC1BAC & This study \\
pYJF27 & Nrc-5F and 3F in pYJF24 & This study \\
pYJF28 & 7101-5F and 3F in pYJF26 & This study \\
pYJF31 & Nrc-Cluster in pYJF27 & This study \\
\hline
\end{tabular}


at $37^{\circ} \mathrm{C}$. For the cleavage of linearized pX330, $400 \mathrm{ng}$ of DNA, pX330-sgRNA ( $\left.30 \mathrm{nM}\right)$ and Cas9 Nuclease (30 nM) were added in the cleavage reaction system to a total reaction volume of $30 \mu$ l. The cleavage reaction was carried out at $37^{\circ} \mathrm{C}$ for $2 \mathrm{~h}$ and overnight, respectively. The cleavage efficiency and off-target effects were assessed using pulsed-field gel electrophoresis (PFGE).

For the cleavage of circular pX330, 400 ng of circular pX330 DNA, different amounts of Cas 9 and sgRNA (mole ratio 1:1) at final concentration $(15,30,60$, and $120 \mathrm{nM})$ were added into the reaction mixture to a total reaction volume of $30 \mu \mathrm{l}$. After incubation, the samples were used to assess the cleavage efficiency and off-target effects using agarose gel electrophoresis.

For the cleavage of HMW plasmid pYPZ37, pYPZ37-sgRNA1 (30 nM), pYPZ37-sgRNA2 (30 nM) and Cas9 Nuclease $(60 \mathrm{nM})$ and different amounts of DNA at final concentration $(50,100,200,500$, and 1,000 ng) were added into the reaction mixture to a total reaction volume of $50 \mu$ l. The cleavage efficiency and off-target effects were assessed using PFGE.

The PFGE was performed with Lambda DNA-Mono Cut Mix as marker in 1\% Megabase agarose gel in 0.5x TBE buffer with the Bio-Rad CHEF Mapper XA System by loading the gel with agarose plugs (or $10 \mu$ l purified plasmids). The CHEF Mapper XA System was set to auto-algorithm program with $5-250 \mathrm{~kb}$ parameters $(6 \mathrm{~V} / \mathrm{cm}$, $0.22-21.79 \mathrm{~s}, 15 \mathrm{~h} 16 \mathrm{~min}, 120^{\circ}$, circulation at $14^{\circ} \mathrm{C}$ ), and the gel was strained by GoldView for visualization using standard gel imaging systems.

\section{Preparation of Fungal DNA}

In this study, fungal strains $N$. fischeri and $P$. fici were cultivated at $25^{\circ} \mathrm{C}$ on potato dextrose agar medium (PDA) for 3 days. For the cleavage reaction in vitro and in vivo, fungal genomic DNA was extracted from lyophilized mycelia by phenol: chloroform: isoamyl alcohol (25:24:1, $\mathrm{pH}>7.8)$ as described previously [28].

\section{Construction of Shuttle pCC1BAC Vectors}

The pyrG and ribo auxotrophy markers were amplified from $A$. fumigatus genomic DNA by using primer pairs Nrc-PyrG-F/R and riboWA-F/R, while the 5' and 3' regions of the $w A$ gene were amplified from A. nidulans genomic DNA by using primers wAup-Nrc-F/R. All three DNA fragments for in vivo yeast recombination contained a minimum of $35 \mathrm{bp}$ overlapping bases with the flanking fragments. The terminal sequence overlaps (up to $1 \mathrm{~kb}$ ) of the target gene clusters (Nrc and Pfma) were prepared by PCR amplification. The PCR primers consisted of an $\sim 25$ bp sequence that annealed to the vector template and an $\sim 25$ bp overhang that overlapped with one end of the target gene cluster. Then these terminal sequence overlaps were ligated to shuttle pCC1BAC vectors by the yeast homologous recombination method to obtain constructs pYJF27and pYJF28. For in vivo Cas9 cleavage application, these two constructs were linearized by NheI and BamHI for further transformation.

\section{In Vitro Cas9 Endonuclease Cleavage for Fungal Genomic DNA}

The reactions were performed as in vitro Cas 9 endonuclease cleavage assays for plasmids. For the cleavage of $N$. fischeri gDNA, 400ng gDNA, Nrc-sgRNA1 (30 nM), Nrc-sgRNA2 (30 nM) and Cas9 endonuclease (60 nM) were added into the cleavage reaction system to a total reaction volume of $100 \mu \mathrm{l}$. For the cleavage of $P$. fici gDNA, 7101-sgRNA1 (30 nM), 7101-sgRNA2 (30 nM), Cas9 endonuclease $(60 \mathrm{nM})$ and different amounts of DNA at final concentrations $(1,2,5,10$, and $20 \mu \mathrm{g})$ were added into the reaction assay to a total reaction volume of $100 \mu \mathrm{l}$. The obtained DNA was concentrated by ethanol precipitation method [27].

Afterwards, all of the gDNA cleaved by Cas9, $1 \mu \mathrm{g}$ of the linearized shuttle pYJF27 or pYJF28, $200 \mu \mathrm{l}$ suspension of freshly prepared yeast (BJ5464-NpgA) competent cells $\left(\sim 10^{8}\right)$ and $600 \mu$ l solution III (Zymo Research, USA) were added into the assembled reaction. The homologous recombination in yeast was performed as the standard manuscript (Zymo Research). Further colonies were verified by extracting their plasmids from SDCt media and subsequent PCR by using diagnostic primers Nrc F5/R5 (Table S1). Plasmid isolation from yeast transformants was performed by using a Zymoprep Kit (Yeast Plasmid Miniprep I, Zymo Research). Plasmids with more amount were further extracted from $E$. coli transformants by PlasmidMini Kit I (Omega, USA) and verified by restriction enzyme digestion (KpnI and NcoI).

\section{Preparation of High-Molecular-Weight (HMW) DNA}

P. fici protoplasts were prepared as described previously [29]. According to previous studies [14] on preparing low melting agarose plugs of HMW DNA, the pellet protoplasts $\left(\sim 5 \times 10^{8}\right)$ were resuspended with the HMW DNA preparation buffer to a total volume of $0.6 \mathrm{ml}$. An equal volume of $1 \%$ low melting agarose was then added to the buffer and incubated at $45^{\circ} \mathrm{C}$. This was sufficient to make 10 plugs (about $100 \mu \mathrm{l}$ per plug) which solidified at $4^{\circ} \mathrm{C}$. The plugs were then incubated in $1 \mathrm{ml}$ of lysis buffer/plug (0.5 M EDTA (pH 9.0), 1\% lauryl sarcosine, $1 \mathrm{mg} / \mathrm{ml}$ proteinase $\mathrm{K}$ ) at $50^{\circ} \mathrm{C}$ for $48 \mathrm{~h}$. Finally, the plugs were extensively washed in 10-20 volumes of the following buffers for one hour in each wash: once with buffer $1\left(0.5 \mathrm{M} \mathrm{EDTA}[\mathrm{pH} 9.0-9.3]\right.$ at $\left.50^{\circ} \mathrm{C}\right)$, once with buffer $2(0.05 \mathrm{M} \mathrm{EDTA}$ [pH 8.0] on ice), three times with buffer 3 (ice-cold TE plus $0.1 \mathrm{mM}$ phenylmethyl sulfonyl fluoride (PMSF) on ice), three times with buffer 4 (ice-cold TE on ice) and finally all plugs were stored in TE buffer at $4^{\circ} \mathrm{C}$.

\section{In-Gel Cas9 Digestion}

In-gel Cas9 digestion was performed as described previously [27]. For the in-gel Cas 9 cleavage of each target sequence, six agarose blocks were used. Additional wash with $0.1 \times$ wash buffer (diluted from $1 \times$ wash buffer with ultrapure water) was immediately performed before Cas9 digestion for diluting the EDTA. After discarding the wash buffer, $100 \mu \mathrm{l}$ of $1 \times$ cleavage buffer (diluted from $5 \times$ cleavage buffer) was added into each tube and the 
agarose blocks equilibrated at room temperature for $30 \mathrm{~min}$. During the equilibration, the assay of Cas9 with sgRNAs was assembled by mixing $60 \mu \mathrm{l}$ of $5 \times$ cleavage buffer, $15 \mu \mathrm{l}$ of DTT $(10 \mathrm{mM}), 30 \mu \mathrm{l}$ of Pfma-sgRNA1 (300 ng/ $\mu \mathrm{l}), 30 \mu \mathrm{l}$ of Pfma-sgRNA2 (300 ng/ $\mu \mathrm{l}), 3 \mu \mathrm{l}$ of Cas9 $(20 \mu \mathrm{M})$ and RNase-free water to a total volume of $300 \mu \mathrm{l}$ and incubated at $37^{\circ} \mathrm{C}$ for $20 \mathrm{~min}$. Subsequently, $50 \mu \mathrm{l}$ of the prepared cleavage solution was added into each micro centrifuge tube (the $1 \times$ cleavage buffer was discarded) and incubated at $37^{\circ} \mathrm{C}$ for $2 \mathrm{~h}$ allowing the diffusion and digestion by Cas 9 to occur in the agarose matrix. Then, the cleavage solution was discarded and all of the available blocks for each target sequence were transferred into a clean $1.5 \mathrm{ml}$ micro centrifuge tube. The plugs were washed with $1 \mathrm{ml}$ of $0.1 \times$ wash buffer and then suspended in $500 \mu \mathrm{l}$ of $1 \times$ GELase buffer (diluted from $50 \times$ GELase buffer with ultrapure water) for $1 \mathrm{~h}$ incubation at room temperature. After discarding the $1 \times$ GELase buffer, the agarose blocks were thoroughly melted by incubating them in a $70^{\circ} \mathrm{C}$ water bath for $5 \mathrm{~min}$. The consistency and appearance of the agarose were assessed after gently flicking the micro centrifuge tube. When the sample has turned into liquid, it can be transferred into a micro centrifuge tube containing the molten agarose into a $45^{\circ} \mathrm{C}$ water bath and equilibrated for $10 \mathrm{~min}$. Finally, $1 \mu \mathrm{l}$ of GELase enzyme was slowly added to the agarose and stirred with a $10 \mu \mathrm{l}$ tip very gently to avoid shearing of the long DNA fragments for further incubation at $45^{\circ} \mathrm{C}$ for $30 \mathrm{~min}$.

After GELase digestion, the sample was cooled to room temperature for $5 \mathrm{~min}$ and flicked gently to examine the appearance of the agarose. If the sample remains liquid, an equal volume $(\sim 170 \mu \mathrm{l})$ of $5 \mathrm{M} \mathrm{CH}_{3} \mathrm{COO}\left(\mathrm{NH}_{4}\right)_{2}$ was added to the digested agarose and gently inverted several times to mix thoroughly. Then, $4 \times$ volume $(\sim 680 \mu \mathrm{l})$ of room-temperature ethanol was added to the micro centrifuge tube and gently inverted several times to mix thoroughly. After incubation at room temperature for $2 \mathrm{~h}$, the DNA was collected as pellet by centrifugation at $12,000 \mathrm{~g}$ at room temperature for $15 \mathrm{~min}$. After washing with $70 \%$ ethanol twice, $20 \mu \mathrm{l}$ of ultrapure water was added into the pellet.

\section{Results}

\section{Design of In Vitro CRISPR/Cas9 Cleavage System}

To build the CRISPR/Cas9 system in vitro for cleaving the fungal BGCs (Fig. 1), we first introduced the coding sequence of Cas 9 endonuclease into $E$. coli for protein overproduction. Based on the information provided by the codon usage database (http://www.kazusa.or.jp/codon/), the ORF of Cas9 gene was amplified from the vector pX330 and subsequently ligated to protein expression vector pET-28a(+) to give pYJF22 (Fig. S1A in the Supplementary Information (SI)). The recombinant His6-tagged protein was purified to near homogeneity as confirmed on SDSPAGE, revealing that its molecular weight was consistent with the predicted value of $164.5 \mathrm{kDa}$ (Fig. S1B).

The CRSIPR design tool (http://crispr.mit.edu/) was used to identify CRISPR guide sequences within the two $1 \mathrm{~kb}$ regions beginning with the $250 \mathrm{bp}$ nearest the hooks (Fig. S2A). As an in vitro transcription based on the T7 RNA polymerase was later used, the CRISPR guide sequence had to conform to the rules governing efficient and precise initiation of the T7 promoter. In detail, the CRISPR guide sequence must start with a guanine $(\mathrm{G})$, followed by a purine (G/A) with ' $G$ ' being significantly more preferred and finally an optional third 'G. Thus, the CRISPR guide sequence should start with ' 5 -GGN', implying the difficulty to obtain a CRISPR guide sequence. Thus, one additional ' $G$ ' was added to the 5 ' end of a 20 bp CRISPR guide sequence according to a previous strategy by Jiang [21]. As mentioned above, an RNA-guided CRISPR/Cas9 system was designed to give several groups of Cas9sgRNA for further cleavage application.

\section{Target Cleavage of Linearized or Circular dsDNA by CRISPR/Cas9}

As a proof-of-principle, we proceeded to apply RNA-guided Cas9 endonuclease in the following cleavage of linearized or circular dsDNA in vitro based on the principle of the CRISPR/Cas9 system. The effector complex, termed Cas9-sgRNA complex, was constituted before cleavage by incubating Cas9 endonuclease with the in vitro transcribed sgRNA. Referring to previous study, it is essential to keep the molar ratio of Cas 9 and sgRNA per target

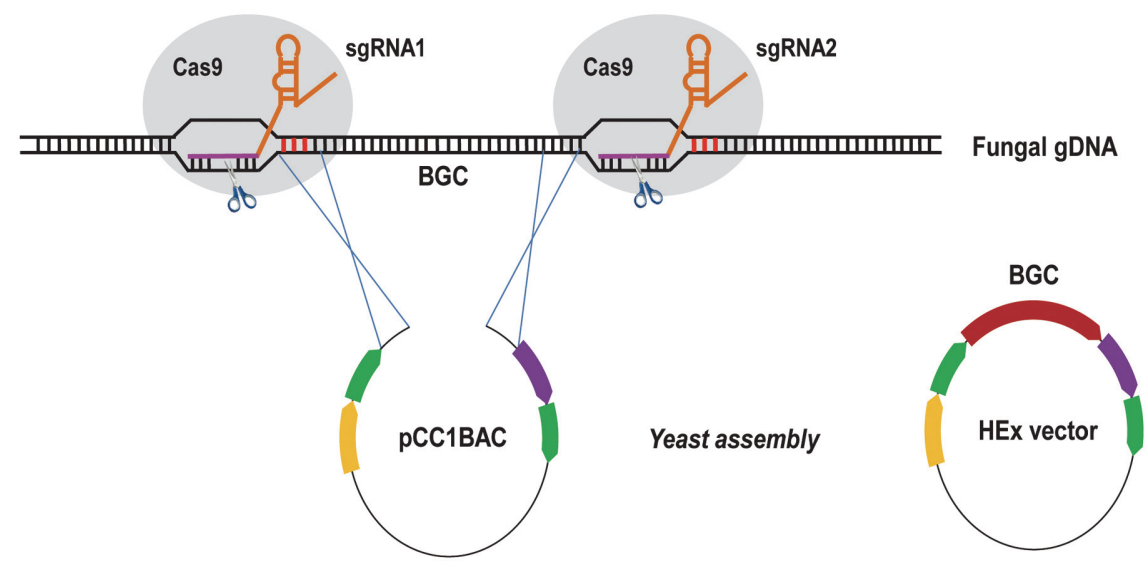

Fig. 1. Outline of in vitro CRISPR/Cas9 cleavage system capturing entire gene clusters in fungi. The genomic DNA extracted from fungi could be cleaved by RNA-guided Cas9 nuclease in vitro, and the target gene clusters are directly linked to heterologous expression vectors. 
PAM

A 5'-TGCTGCCAGTGGCGATAAGTCGTGTCTTACCGGGTTGGAC-3' 3'-ACGACGGTCACCGCTATTCAGCACAGAATGGCCCAACCTG-5'

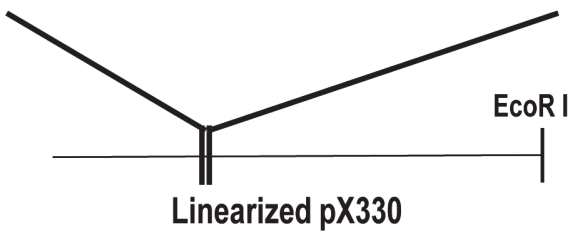

PAM

B

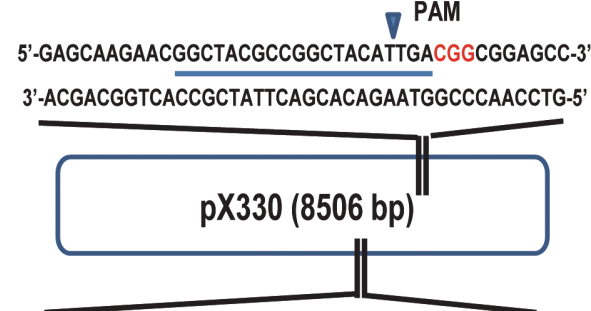

،

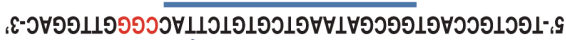
PAM $\triangle$

\section{C}

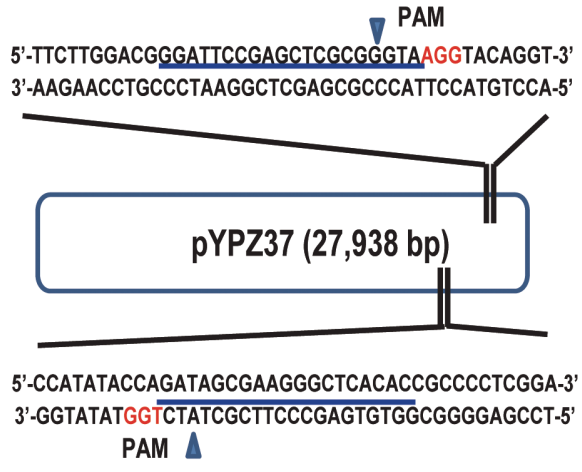

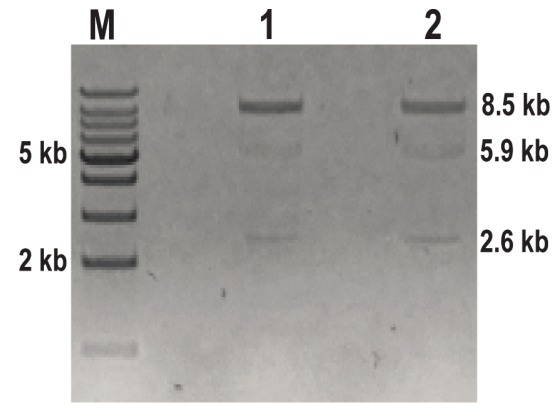

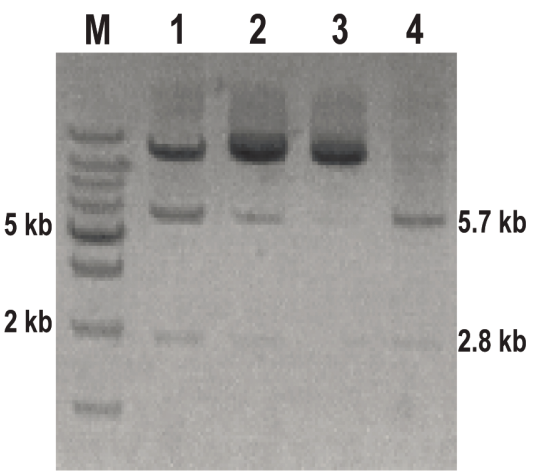

M $12 \begin{array}{llll}2 & 3 & 4 & 5\end{array}$

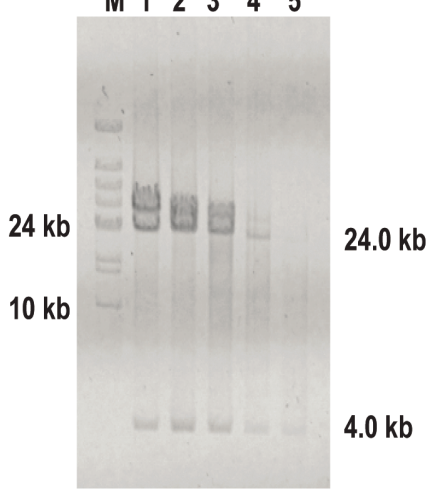

Fig. 2. Cleavage of linearized or circular dsDNA by CRISPR/Cas9 in vitro. (A) Designed sgRNA and linearized pX330 by EcoRI $(200 \mathrm{ng} / \mu \mathrm{l}, 8.5 \mathrm{~kb})$ cleaved by Cas 9 endonuclease. Lane M: marker; Lanes 1 and 2: linearized pX330 cleaved by Cas 9 at $37^{\circ} \mathrm{C}$ for $2 \mathrm{~h}$ and overnight, respectively. (B) Designed sgRNAs and circular pX330 (200 ng/ $\left.\mu \mathrm{l}, 8.5 \mathrm{~kb}\right)$ cleaved by Cas 9 endonuclease. Lane M: marker; Lanes 1-4: circular pX330 cleaved by Cas9 and sgRNA at different concentrations of $60 \mathrm{nM}$, $30 \mathrm{nM}, 15 \mathrm{nM}$ and $120 \mathrm{nM}$. (C) Designed sgRNAs and circular pYPZ37 (415 ng/ $\mu \mathrm{l}, 28.0 \mathrm{~kb}$ ) cleaved by Cas 9 endonuclease. Lane M: marker; Lane 1-5: circular pYPZ37 cleaved by Cas9 at different amounts of 1,000 ng, $500 \mathrm{ng}, 200 \mathrm{ng}, 100 \mathrm{ng}$, and $50 \mathrm{ng}$.

site at 10:10:1 or higher to obtain the best cleavage efficiency [30]. Subsequently, one protospacer (S1) on the linearized plasmid pX330 by EcoRI was selected to transcribe pX330-sgRNA1. When pX330-sgRNA1 was complexed with Cas 9 , the linearized pX330 $(8.5 \mathrm{~kb})$ was cleaved at the predicted position to generate two dsDNA fragments whose lengths were $5.9 \mathrm{~kb}$ and $2.6 \mathrm{~kb}$, respectively (Fig. 2A). The gel electrophoresis analysis of pX330 cleavage samples indicated that overnight digestion was more efficient, although the linearized plasmid pX330 had been cut in two hours. Therefore, the overnight incubation time was used in the following assays.

To investigate the cleavage reaction of two sgRNAs complexed with Cas9, two protospacers (S1 and S2) on pX330 were selected to transcribe pX330-sgRNA1 and pX330-sgRNA2. Incubation of the circular pX330 (8.5 kb) DNA with Cas9 endonuclease, pX330-sgRNA1 as well as pX330-sgRNA2 led to the cleavage at the predicted position, yielding two fragments with sizes of $5.7 \mathrm{~kb}$ and $2.8 \mathrm{~kb}$ (Fig. 2B). Further incubation of Cas 9 with sgRNAs (mole ratio 1:1) was carried out at different final concentrations (15, 30, 60 and $120 \mathrm{nM}$ ). Clear bands at the expected lengths were observed in each of the four lanes, but with higher digestion efficiency at higher concentration. Surprisingly, the circular pX330 was completely cleaved into dsDNA fragments at the final concentration of $120 \mathrm{nM}$. Thus, a high final concentration of Cas9 and sgRNA is essential for the CRISPR/Cas9 in vitro cleavage system.

To investigate the cleavage efficiency for dsDNA with high molecular weight (HMW) by CRISPR/Cas9, we 
proceeded with the cleavage assay of the circular plasmid pYPZ37 $(28.0 \mathrm{~kb})$ [25]. In analogy to the incubation of pX330-sgRNAs, two protospacers (S1 and S2) on pYPZ37 were selected to transcribe pYPZ37-sgRNA1 and pYPZ37-sgRNA2. When pYPZ37-sgRNA1 and pYPZ37-sgRNA2 were complexed with Cas9, the circular pYPZ37 was cleaved at the predicted position to generate two fragments with sizes of $24.0 \mathrm{~kb}$ and $4.0 \mathrm{~kb}$, respectively (Fig. 2C). Incubation with different amounts of DNA (50, 100, 200, 500, and 1,000 ng) were further carried out, indicating more cleaved product obtained with higher amount of DNA by PFGE. This proved the sufficient cleavage efficiency of the RNA-guided Cas9 system in vitro, even for high amounts of DNA with high molecular weight. Taken together, the in vitro CRISPR/Cas9 system can accurately cut linear or circular dsDNA, and the final concentrations of dsDNA, Cas9 and sgRNA will affect the cleavage efficiencies.

\section{Capturing Entire Fungal BGCs with CRISPR/Cas9 System}

Having identified the in vitro CRISPR/Cas9 cleavage system, it was then applied to manipulate the fungal genomic DNA. Two gene clusters, named $N r c$ and $P f m a$, with different lengths were selected to be edited. Nrc gene cluster with a length of $18.0 \mathrm{~kb}$ derived from $N$. fischeri (Fig. 3A) is responsible for the synthesis of neosartoricin B with immunomodulatory activity [11]. Pfma is a DHN melanin biosynthetic gene cluster in P. fici with a length of $52.7 \mathrm{~kb}$ (Fig. 3C) [25]. At first, we constructed E. coli-yeast-Aspergillus shuttle pCC1BAC vectors by using homologous recombination in S. cerevisiae, which enables insertion of fragments with sizes up to a couple of $100 \mathrm{~kb}$ (Figs. 1 and S3). As a result, the acquired constructs pCC1BAC-wA-pyrG-Nrc (pYJF27) and pCC1BACwA-ribo-Pfma (pYJF28) consisted of (1) the linearized pCC1BAC vector, (2) yeast centromere sequence (CEN), autonomously replicating sequence (ARS), and a URA3 marker, (3) a 5' wA -3' wA cassette synthesized by fusion PCR, (4) the pyrG and ribo auxotrophy markers and (5) the terminal sequence overlaps ( 1000 nucleotides) of the target gene clusters.

Subsequently, two sgRNAs necessary for cleaving gene clusters were synthesized in vitro and combined with Cas9-treated fungal genomic DNA. With linearized pYJF27 and pYJF28 by NheI and BamHI, respectively, in vivo recombination in S. cerevisiae between the hooks and Cas9-treated genomic DNAs containing Nrc or Pfma gene cluster was carried out. After 2-3 days of cultivation, two positive colonies harboring a $31.4 \mathrm{~kb}$ fragment were obtained from the mixture containing $1 \mathrm{mg}$ of $N$. fischeri genomic DNA and $1 \mathrm{mg}$ of linearized pYJF27 plasmid by PCR using the diagnostic primer pair Nrc F5/R5 and the following restriction enzyme digestions. Thus, we successfully captured the entire BGC of Nrc by RNA-guided Cas9 system in N. fischeri (Fig. 3B).

A

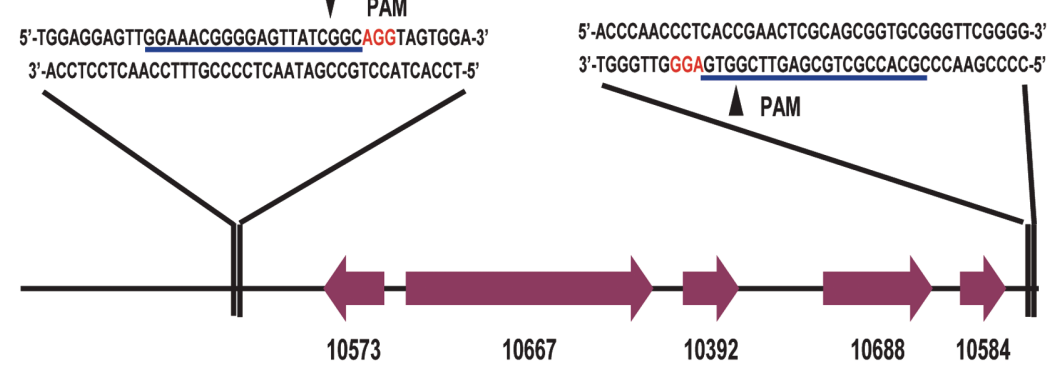

Nrc cluster from N. fischeri (Target: 18.0kb)

C

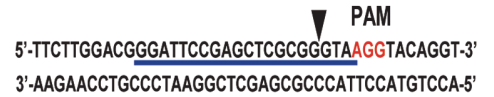

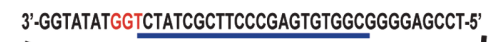

5'-CCATATACCAGATAGCGAAGgGCTCACACCGCCCCTCGGA-3'
3'-AAGAACCTGCCCTAAGGCTCGAGCGCCCATTCCATGTCCA-5

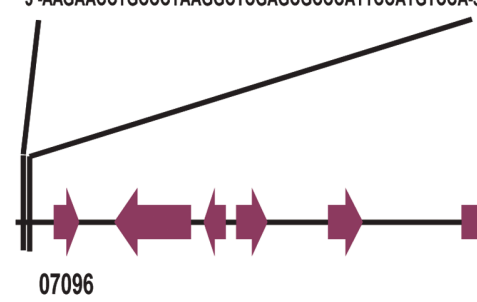

B

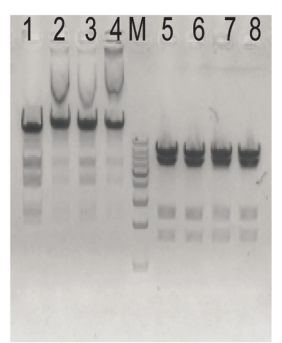

M 12

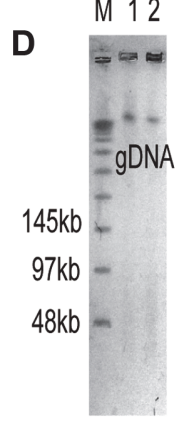

Pfma cluster from P. fici (Target: 52.7kb)

Fig. 3. Capturing the fungal gene cluster with CRISPR/Cas9 cleavage system. (A) SgRNA pairs designed to target Nrc gene cluster from N. fischeri. (B) Verification of the obtained construct pYJF31 containing Nrc gene cluster by restriction endonuclease digestion. Lane M: marker; Lanes 1-4: plasmids digested by KpnI. Lengths of target fragments are 15,631 bp, 6,209 bp, 4,432 bp, 2,782 bp and 2,388 bp; Lane 5-8: plasmids digested by NcoI. Lengths of target fragments are 7,600 bp, 7,348 bp, 6,271 bp, 5,975 bp, 2,511 bp and 1,737 bp. (C) SgRNA pairs designed to target genome Pfma gene cluster from P. fici. (D) Genomic DNA of P. fici analyzed using PFGE. Lane M: marker; Lane 1: P. fici gDNA without Cas9 cleavage; Lane 2: P. fici gDNA cleaved by Cas 9 in low melting agarose plugs. 


\section{Discussion}

In the post-genomic era, the heterologous expression approach has become one of the most effective ways to activate 'silent' gene clusters for mining fungal SM "dark matter" [31,32]. Usually, a fungal genome includes about 20-90 BGCs which span 10-130 kb for each BGC $[1,17]$. Therefore, a bottleneck for successful heterologous expression of a BGC is the efficient cloning of large DNA fragments. Currently, available techniques for large DNA fragment cloning are mostly based on PCR or restriction-enzyme-based methods [33], which are severely constrained by errors, restriction site limitation and low efficiency when a gene cluster spans dozens of kilobases [20]. Though TAR had been developed for the efficient assembly of BGCs from human [15] and bacteria [34], it is still challenging to clone large DNA fragments from fungi. In a pioneer group, Keller lab developed a fungal artificial chromosome (FAC) system that can capture a large DNA fragment (over $100 \mathrm{~kb}$ ). However, this technique is high cost and laborious because it is based on the screening and sequencing of large-scale colonies $[14,17]$. Another limitation is that FAC DNA fragments are randomly selected and cannot define the exact border of a BGC. Recently, the Cas9 as a programmable endonuclease has demonstrated its advantages in precisely editing genomic DNA [35-36]. Based on the CRISPR/Cas9 technology, several in vivo genetic manipulation methods have been established in filamentous fungi [24, 37-39]. However, the application of the CRISPR/Cas9 system in vitro for capturing/editing an entire BGC is limited.

To capture entire BGCs from fungi, we conducted many trials using methods including TAR, and SOE (splicing by overlap extension)-PCR based yeast recombination [40] but failed in the past years. Considering the differences between eukaryotic and prokaryotic cells, we presume that some difficulties are not unknown for manipulating eukaryotic genomic DNA. The advantages of the CRISPR/Cas9 system in gene editing encouraged us to achieve the entire BGCs capturing. When designing the CRISPR/Cas9 system, we first tried to use the CRISPR/Cas9 system to cleave linearized and circular dsDNA in vitro. Among the many possible reasons, a variety of sgRNAs were applied to the CRISPR/Cas9 system, and the cleavage and cloning results suggested that the selection of sgRNAs did not appear to affect the cleavage and cloning efficiency [21]. Using two plasmid DNAs with different sizes of pX330 $(8.5 \mathrm{~kb})$ and pYPZ37 $(28.0 \mathrm{~kb})$ as samples, we found that the two plasmids were digested efficiently as expected (Figs. 2A and 2B). Our CRISPR/Cas9 system revealed sufficient cleavage in vitro, indicating that it can be excluded for restricting the efficiency of the cleavage CRISPR/Cas9 system. The success of the trial in plasmid cleavage encourages us for our next capture of the entire group of BGCs from fungal genomic DNA. The size of the $N r c$ gene cluster is about $18.0 \mathrm{~kb}$ from $N$. fischeri, with the genome size of $32 \mathrm{Mb}$ [41]. By the extraction of genomic DNA in combination with yeast recombination, the entire gene cluster Nrc was target isolated and constructed onto BAC vectors (Figs. 3A and 3B).

To further utilize the in vitro CRISPR/Cas9 cleavage system for larger cluster editing, we attempted to prepare HMW DNA by low melting agarose plugs [14] and in-gel Cas9 digestion [27]. This will avoid the destruction of fungal genomic DNA by phenol: chloroform: isoamyl alcohol. Therefore, the CRISPR/Cas9 cleavage system was extended for editing the fungal strain $P$. fici with the help of PEG-mediated protoplast transformation [29]. The digestion of protoplast was performed according to the preparation of HMW DNA in A. terreus [14]. Subsequently, these treated protoplasts were digested by Cas9-sgRNA complexes and used to assess the cleavage efficiency by PFGE. Although we obtained a relatively high-quality genomic DNA of $P$. fici (Fig. 3D), it is still difficult to isolate the $P f m a$ gene cluster from $P$. fici genome. Correspondingly, the length of the Pfma gene cluster was one thousandth of the total gene length of P. fici genome DNA at $52 \mathrm{Mb}$ [42]. The ratio between the target gene cluster and the fungal genome is a key possible reason for obtaining the target gene cluster. Although we failed to obtain a gene cluster greater than $50 \mathrm{~kb}$, it should be possible to extend the method to clusters larger than this. Therefore, the entire BGC capture efficiency could be improved by procedure optimization like desalting after end repair, using super competent cells and applying electro transformation approaches.

In conclusion, we developed an in vitro CRISPR/Cas9 cleavage system for capturing the target gene clusters from filamentous fungi. Using this system, the linear or circular DNA can be cleaved efficiently. It is a possible replacement for the restriction enzymes due to having no limitation of site/place selection. Ultimately, we captured the entire gene cluster of Nrc from $N$. fischeri. This is a big step for obtaining BGCs precisely without PCR amplification steps from filamentous fungi. Our results provide a new approach to manipulate fungal genomic DNA and an efficient tool for mining fungal BGCs for heterologous expression.

\section{Acknowledgments}

We thank Professors Ling Lu and Chunbo Lou for providing us plasmids pX330 and pCC1BAC. This work was supported by the National Key Research and Development Program of China (2018YFA0900701), Key Research Program of Frontier Sciences, CAS (ZDBS-LY-SM016), The Senior User Project of RV KEXUE (KEXUE2019GZ05) and Construction of the Registry and Database of Bioparts for Synthetic Biology, CAS (ZSYS-016).

\section{Conflict of Interest}

The authors have no financial conflicts of interest to declare.

\section{References}

1. Romsdahl J, Wang CCC. 2019. Recent advances in the genome mining of Aspergillus secondary metabolites (covering 2012-2018). Medchemcomm. 10: 840-866.

2. Araujo R, Sampaio-Maia B. 2018. Fungal genomes and genotyping. Adv. Appl. Microbiol. 102: 37-81.

3. Sharma KK. 2016. Fungal genome sequencing: basic biology to biotechnology. Crit. Rev. Biotechnol. 36: 743-759. 
4. Li W, Yin WB. 2019. Genetic mining of the "dark matter" in fungal natural products. Sci. China Life Sci. 62: 1250-1252.

5. Wang B, Guo F, Dong SH, Zhao H. 2019. Activation of silent biosynthetic gene clusters using transcription factor decoys. Nat. Chem. Biol. 15: 111-114.

6. Keller NP. 2019. Fungal secondary metabolism: regulation, function and drug discovery. Nat. Rev. Microbiol. 17: 167-180.

7. Chiang YM, Oakley CE, Ahuja M, Entwistle R, Schultz A, Chang S-L, et al. 2013. An efficient system for heterologous expression of secondary metabolite genes in Aspergillus nidulans. J. Am. Chem. Soc. 135: 7720-7731.

8. Harvey CJB, Tang M, Schlecht U, Horecka J, Fisher C, Lin H-C, et al. 2018. Hex: a heterologous expression platform for the discovery of fungal natural products. Sci. Adv. 4: eaar5459.

9. Chiang YM, Szewczyk E, Davidson AD, Keller N, Oakley BR, Wang CC, et al. 2009. A gene cluster containing two fungal polyketide synthases encodes the biosynthetic pathway for a polyketide, asperfuranone, in Aspergillus nidulans. J. Am. Chem. Soc. 131: 29652970.

10. Nielsen MT, Nielsen JB, Anyaogu DC, Holm DK, Nielsen KF, Larsen TO, et al. 2013. Heterologous reconstitution of the intact geodin gene cluster in Aspergillus nidulans through a simple and versatile PCR based approach. PLoS One 8: e72871.

11. WB Yin, YH Chooi, Smith AR, Cacho RA, Hu Y, White TC, et al. 2013. Discovery of cryptic polyketide metabolites from dermatophytes using heterologous expression in Aspergillus nidulans. ACS Synth. Biol. 2: 629-634.

12. Lin TS, Chiang YM, Wang CC. 2016. Biosynthetic pathway of the reduced polyketide product citreoviridin in Aspergillus terreus var. Aureus revealed by heterologous expression in Aspergillus nidulans. Org. Lett. 18: 1366-1369.

13. Chiang YM, Ahuja M, Oakley CE, Entwistle R, Asokan A, Zutz C, et al. 2016. Development of genetic dereplication strains in Aspergillus nidulans results in the discovery of aspercryptin. Angew. Chem. Int. Ed. Engl. 55: 1662-1665.

14. Bok JW, Ye R, Clevenger KD, Mead D, Wagner M, Krerowica A, et al. 2015. Fungal artificial chromosomes for mining of the fungal secondary metabolome. BMC Genomics 16: 343.

15. Kouprina N, Larionov V. 2006. Tar cloning: insights into gene function, long-range haplotypes and genome structure and evolution. Nat. Rev. Genet. 7: 805-812.

16. Lee NC, Larionov V, Kouprina N. 2015. Highly efficient CRISPR/Cas9-mediated tar cloning of genes and chromosomal loci from complex genomes in yeast. Nucleic Acids Res. 43: e55.

17. Clevenger KD, JW Bok, Ye R, Miley GP, Verdan MH, Velk T, et al. 2017. A scalable platform to identify fungal secondary metabolites and their gene clusters. Nat. Chem. Biol. 13: 895-901.

18. Doudna JA, Charpentier E. 2014. Genome editing. The new frontier of genome engineering with CRISPR-Cas9. Science 346: 1258096.

19. Hsu PD, Lander ES, Zhang F. 2014. Development and applications of CRISPR-Cas9 for genome engineering. Cell 157: $1262-1278$.

20. Liu Y, Tao W, Wen S, Li Z, Yang A, Deng Z, et al. 2015. In vitro CRISPR/Cas9 system for efficient targeted DNA editing. $m$ Bio 6: e01714-01715.

21. Jiang W, Zhao X, Gabrieli T, Lou C, Ebenstein Y, Zhu TF. 2015. Cas9-assisted targeting of chromosome segments catch enables onestep targeted cloning of large gene clusters. Nat. Commun. 6: 8101.

22. Wang JW, Wang A, Li K, Wang B, Jin S, Reiser M, et al. 2015. CRISPR/Cas9 nuclease cleavage combined with gibson assembly for seamless cloning. Biotechniques 58: 161-170.

23. Zhang C, Lu L. 2017. Precise and efficient in-frame integration of an exogenous gfp tag in Aspergillus fumigatus by a CRISPR system. Methods Mol. Biol. 1625: 249-258.

24. Zhang C, Meng X, Wei X, Lu L. 2016. Highly efficient CRISPR mutagenesis by microhomology-mediated end joining in Aspergillus fumigatus. Fungal Genet. Biol. 86: 47-57.

25. Zhang P, Wang X, Fan A, Zheng Y, Liu X, Wang S, et al. 2017. A cryptic pigment biosynthetic pathway uncovered by heterologous expression is essential for conidial development in pestalotiopsis fici. Mol. Microbiol. 105: 469-483.

26. Cacho RA, Jiang W, Chooi YH Walsh CT, Tang Y. 2012. Identification and characterization of the echinocandin B biosynthetic gene cluster from Emericella rugulosa NRRL 11440. J. Am. Chem. Soc. 134: 16781-16790.

27. Jiang W, Zhu TF. 2016. Targeted isolation and cloning of 100-kb microbial genomic sequences by cas9-assisted targeting of chromosome segments. Nat. Protoc. 11: 960-975.

28. Xu X, Liu L, Zhang F, Wang W, Ki J, Guo L, et al. 2014. Identification of the first diphenyl ether gene cluster for pestheic acid biosynthesis in plant endophyte pestalotiopsis fici. Chembiochem 15: 284-292.

29. Wu G, Zhou H, Zhang P, Wang W, Li J, Gou L, et al. 2016. Polyketide production of pestaloficiols and macrodiolide ficiolides revealed by manipulations of epigenetic regulators in an endophytic fungus. Org. Lett. 18: 1832-1835.

30. Anders C, Jinek M. 2014. In vitro enzymology of cas9. Methods Enzymol. 546: 1-20.

31. Alberti F, Foster GD, Bailey AM. 2017. Natural products from filamentous fungi and production by heterologous expression. Appl. Microbiol. Biotechnol. 101: 493-500.

32. Qiao Y-M, Yu R-L, Zhu P. 2019. Advances in targeting and heterologous expression of genes involved in the synthesis of fungal secondary metabolites. RSC Adv. 9: 35124-35134.

33. Cobb RE, Zhao H. 2012. Direct cloning of large genomic sequences. Nat. Biotechnol. 30: 405-406.

34. Kouprina N, Noskov VN, Larionov V. 2020. Selective isolation of large segments from individual microbial genomes and environmental DNA samples using transformation-associated recombination cloning in yeast. Nat. Protoc. 15: 734-749.

35. Jiang W, Bikard D, Cox D, Zhang F, Marraffini LA. 2013. RNA-guided editing of bacterial genomes using CRISPR-Cas9 systems. Nat. Biotechnol. 31: 233-239.

36. Karvelis T, Gasiunas G, Siksnys V. 2013. Programmable DNA cleavage in vitro by cas9. Biochem. Soc. Trans. 41: 1401-1406.

37. Huang MY, Mitchell AP. 2017. Marker recycling in Candida albicans through CRISPR-Cas9-induced marker excision. $m S p h e r e ~ 2: 2$.

38. Krappmann S. 2017. CRISPR-Cas9, the new kid on the block of fungal molecular biology. Med. Mycol. 55: 16-23.

39. Sarkari P, Marx H, Blumhoff ML, Mattanovich D, Sauer M, Steiger MG. 2017. An efficient tool for metabolic pathway construction and gene integration for Aspergillus niger. Bioresour. Technol. 245: 1327-1333.

40. Muller H, Annaluru N, Schwerzmann JW, Rhichardson SM, Dymond J, Cooper EM, et al. 2012. Assembling large DNA segments in yeast. Methods Mol. Biol. 852: 133-150.

41. Wortman JR, Fedorova N, Crabtree J, Jordar V, Maiti BJ, Amedeo P, et al. 2006. Whole genome comparison of the A. fumigatus family. Med. Mycol. 44: S3-S7.

42. Wang X, Wu F, Liu L, Liu X, Che Y, Keller NP, et al. 2015. The bzip transcription factor pfzipa regulates secondary metabolism and oxidative stress response in the plant endophytic fungus pestalotiopsis fici. Fungal Genet. Biol. 81: 221-228. 